\title{
A simplified model of gas turbine unit adaptive control
}

\author{
B. V. Kavalerov ${ }^{1}$, I. R. Ziyatdinov ${ }^{1, *}$, and S. D. Basargin ${ }^{1}$ \\ ${ }^{1}$ Perm National Research Polytechnic University, Perm, Russia
}

\begin{abstract}
The article explores the possibilities of building automatic control systems for gas turbine power plants. The use of the principles of adaptive control with a reference model and a signal effect is being studied. As an adaptation method, a method based on the Lyapunov function is used, in order to reduce the time for searching for an extremum. There is a need to ensure the specified indicators of electricity the quality in a constantly changing electrical load and changes in operating modes of the power plant in gas turbine units. The article presents a model of a gas turbine installation for the study of adaptive control. It is shown that the improvement of one coordinate is possible to obtain due to some deterioration of the other coordinate. For further study of this problem, a simplified second-order dynamic model has been constructed. With the help of such a model, complex studies of various stages of adaptive control were carried out. Results were obtained by varying the parameters of adaptive control and simulation parameters. The research results are designed to find the optimal ratios of adaptive automatic control systems parameters for power industry gas turbine plants.
\end{abstract}

\section{Introduction}

Aviation gas turbine engines (GTE) are used not only for aircraft. Gas turbine units (GTU) are also being created on the basis of aviation gas turbine engines to generate electrical energy. Managing such GTUs is not an easy task. As an object of control, a GTU is a nonlinear system with non-stationary parameters. Therefore, adaptive management of the gas turbine plant has good prospects; a large number of publications are devoted to this topic [1-3]. But the use of adaptive control requires serious research to assess its capabilities and limitations in the management of a gas turbine various modes [414]. The main method of research in this case due to the need to cover a wide range of regime situations is mathematical modeling.

\section{Model of gas turbine unit}

Consider a simplified model of a GTU, its structure is shown in Figure 1 The figure indicates: FT- is a free turbine, TC - is a turbocharger, $\mathrm{NU}$ - is a non-linear unit, $n_{\mathrm{FT}}-$ is the frequency of rotation of a free turbine, $n_{\mathrm{TC}}-$ is the frequency of rotation of the turbocharger, $G_{\mathrm{F}}$ - is fuel consumption, $N_{\mathrm{E}}$ - is available power of the free turbine, $N_{\mathrm{G}}$ - power of the electric generator.

GTU is operable only if there is an automatic control system (ACS). Figure 2 shows a simplified scheme of ACS with feedback on $n_{F T}$.

\section{Adaptive control with reference model}

Adaptive control of electromechanical systems with elastic couplings is considered in [15]. The audit showed that these algorithms work well with the control of a gas turbine [4]. Consider an algorithm with a reference model. Consider the effect of $z$, which is fed to the input of the ACS and corrects its behavior in order to bring this behavior closer to the behavior of the reference model [16]:

$$
\mathbf{z}(t)=-h \operatorname{sgn}\left(\mathbf{B}^{\mathrm{T}} \mathbf{P e}\right) ; h>0,
$$

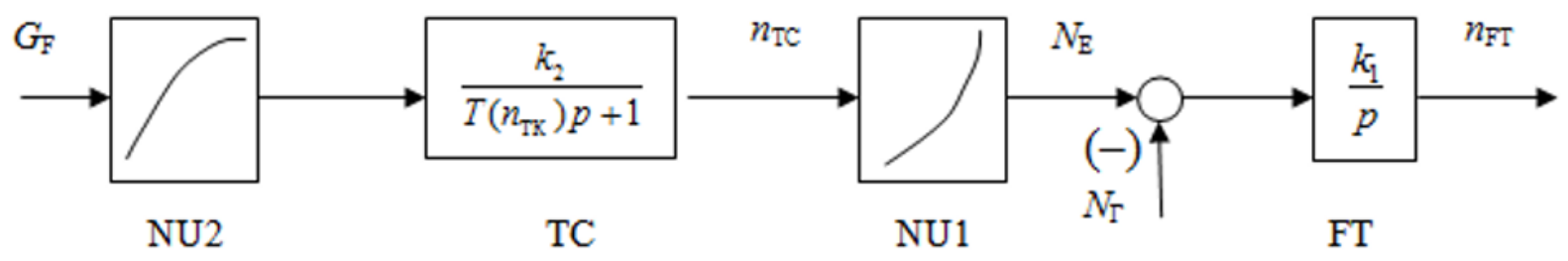

Fig. 1. Block diagram of a nonlinear model GTU.

\footnotetext{
* Corresponding author: i.ziyatdinoff@mail.ru
} 


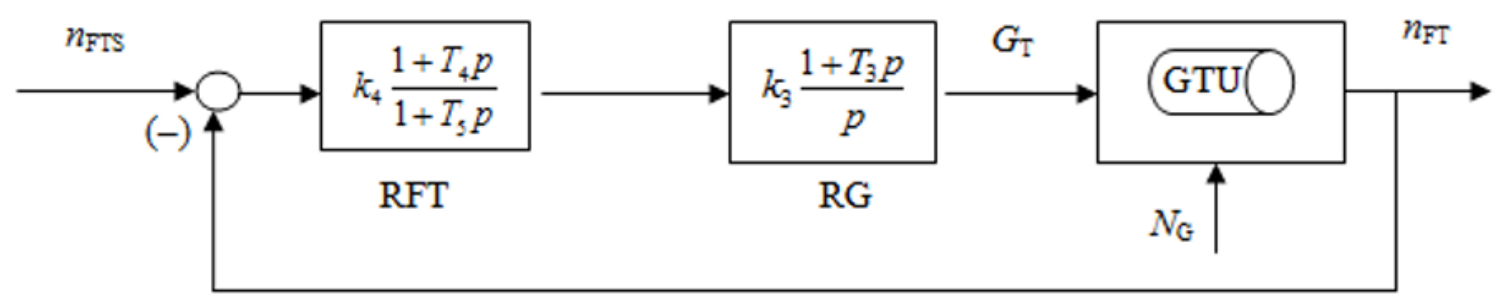

Fig. 2. Block diagram of the model of ACS GTU: GTU - model of GTU according to Figure 1, RFT - free turbine regulator, RG - gas regulator, $n_{\text {FTS }}-$ free turbine speed reference $n_{\text {FT. }}$.

where $\left(\operatorname{sgn} \mathbf{B}^{\mathrm{T}} \mathbf{P e}\right)_{i}=\operatorname{sgn}\left(\mathbf{B}^{\mathrm{T}} \mathbf{P e}\right)_{i}$. Matrix $\mathbf{P}$ is a solution to the matrix equation:

$$
\mathbf{P} \mathbf{A}^{\mathrm{T}}+\mathbf{A}^{\mathrm{T}} \mathbf{P}=-\mathbf{Q}
$$

where the matrix $\mathbf{Q}$ is recommended to choose a diagonal ( $>0$ ). Matrices $\mathbf{A}$ and $\mathbf{B}$ describe the model presented in Figure 2. The vector $\mathbf{e}$ is a four-dimensional error vector, that is, the differences between the outputs of the model presented unit integrators in Figure 2 and the reference model. The condition of the Lyapunov function negative definiteness with respect to the error $\mathbf{e}$ is satisfied if the value of the parameter $h$ is chosen as

$$
h \geq M_{\phi}\left\|\mathbf{B}_{t}^{+}\right\|
$$

what is guaranteed $\lim _{t \rightarrow \infty} \mathrm{e}(t)=0$.

The reference model is chosen the same structure as the model presented in Figure 2, but with constant parameters, linearization is performed at the point of the nominal mode. The adjustment of the regulators is close to the so-called setting for a «symmetric optimum» $[17,18]$.

\section{Experimental results}

Modeling has shown that the improvement in regulatory quality is insignificant. Figure 4 shows $n_{\mathrm{FT}}$ transients when the generator power drops from 2.5 MW to $2 \mathrm{MW}$. For $h=10,000$, the transient process is closer to the reference model than for $h=500$. However, the transient processes in the other variables are accompanied by strong oscillations around the desired trajectory. Such oscillations may be unacceptable for GTU; to eliminate them, the sign function can be replaced by sigmoid $[19,20]$. The transients shown in Fig. 4 were obtained for the GTU model with non-linearities of type $\left(T\left(n_{\mathrm{TC}}\right)\right)$, non-linearities of type NB are turned off, and if they are connected, the situation worsens even more. The reason for this is that the circuit shown in Figure 3 tries to eliminate all 4 errors at the expense of a single signal effect $z$.

If we leave only one error signal $e 1$, then for the first variable $n_{\mathrm{FT}}$ we get almost complete coincidence with the reference model even if all non-linearities are taken into account in the ACS model of the GTU, however, other variables are not tuned at all. To study this, we consider the model of the simplest dynamic system of the second order.

\section{The dynamic model of the second order}

Take the model shown in Figure 4. $K_{r}-$ this is the regulator (gain). Nonlinearity will be simulated by changing the coefficient $K_{d}$. The model shown in Figure 4 with $K_{d}=1$ is taken as the reference model. The figure
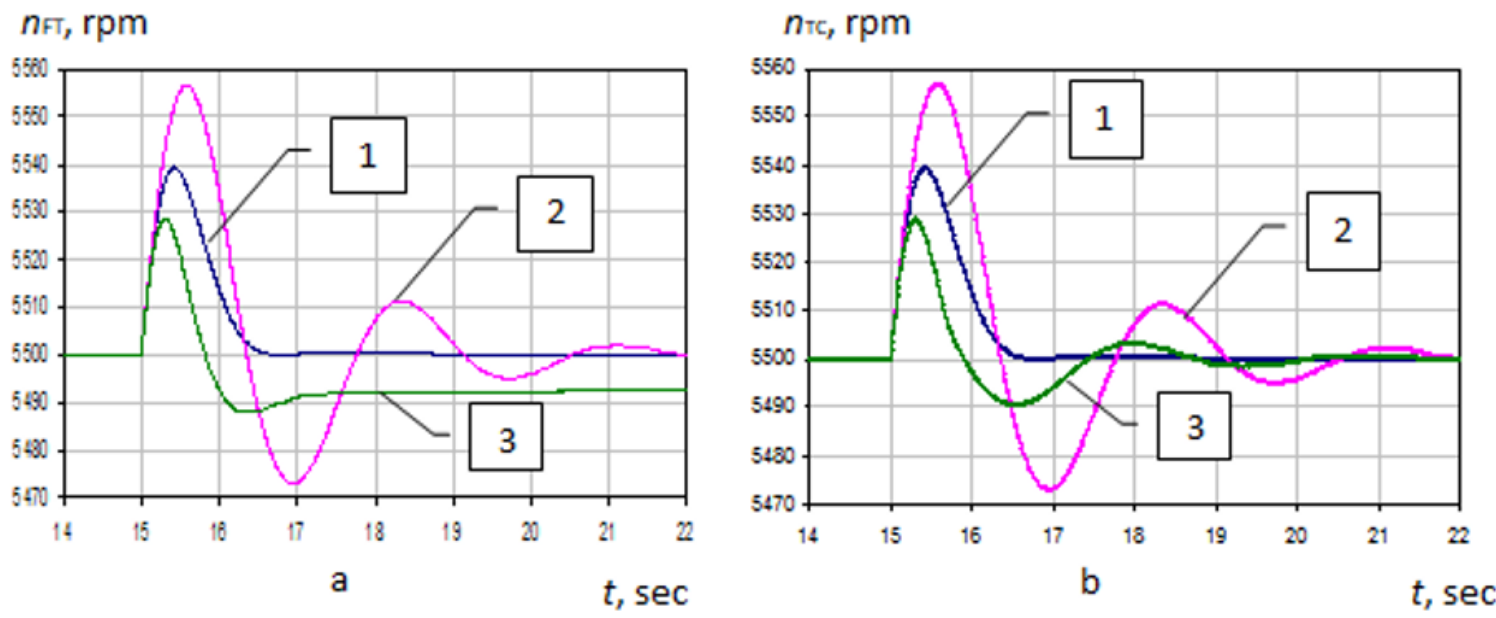

Fig. 3. Transition by $n_{\text {Ст: }}$ a $-h=500, \sigma-h=10000 ; 1-$ reference model, 2 - ACS GTU without adaptive control, 3 -with adaptive control. 


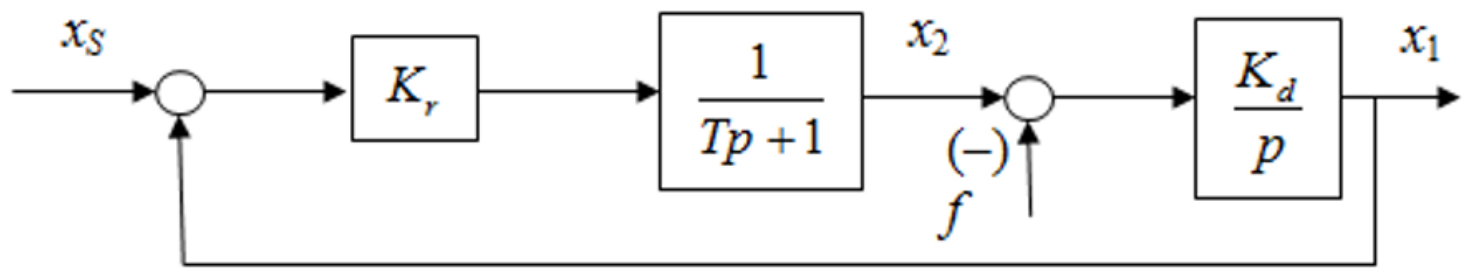

Fig. 4. Second order dynamic system.

shows: $f$ - disturbing effect, $x_{1}$ and $x_{2}$ - variables, by analogy with the model of ACS GTU we assume that they have the dimension: revolutions per minute.

Experiments have shown that when $K_{d}$ is varied, the largest error appears near the exact value ( $K_{d}$ value of the reference model), Figure 5. The simulation was performed at $h=100$, the integration step of the model differential equations is $d t=0.00001$. The most accurate result is given by the simplest Euler method for solving differential equations of the model.

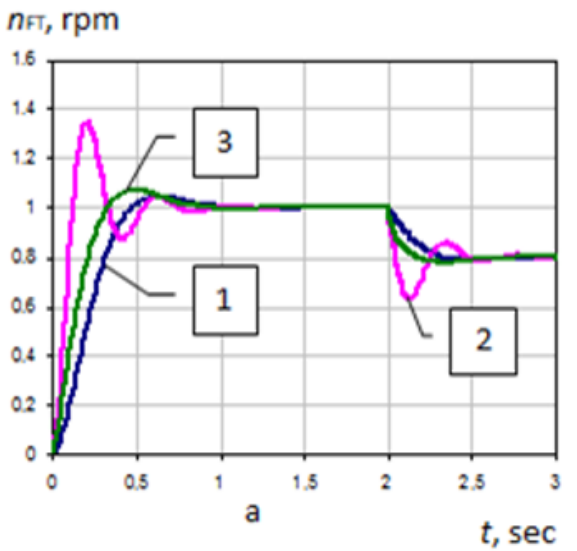

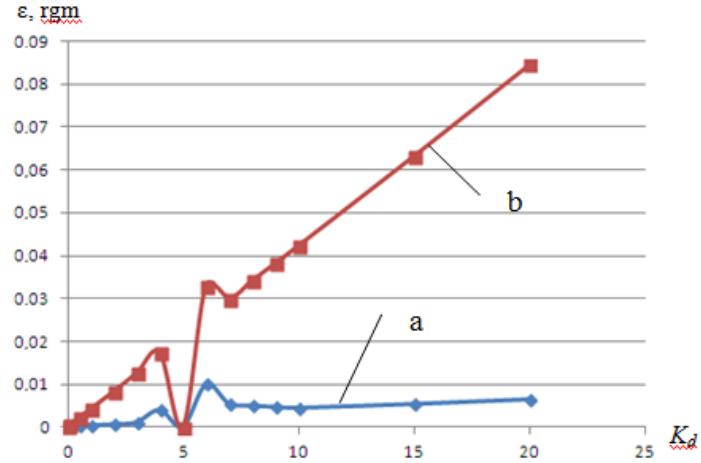

Fig. 5. Error dependence on the model parameter deviation: a Euler method, $\mathrm{b}$ - Runge-Kutta method, calculation step $-d t=$ $0.00001 \mathrm{~s}$.

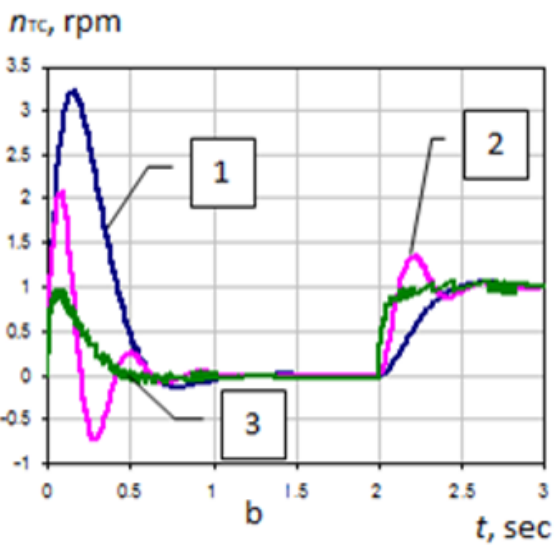

Fig. 6. Transition on $\mathrm{n}_{\mathrm{FT}}$ and $n_{\mathrm{TC}}$ with the first matrix $\mathbf{Q}: \mathrm{a}-\mathrm{n}_{\mathrm{FT}}, \mathrm{b}-\mathrm{n}_{\mathrm{TC}} ; 1$ - reference model, 2 - ACS GTU without adaptive control, 3 - ACS GTU with adaptive control.
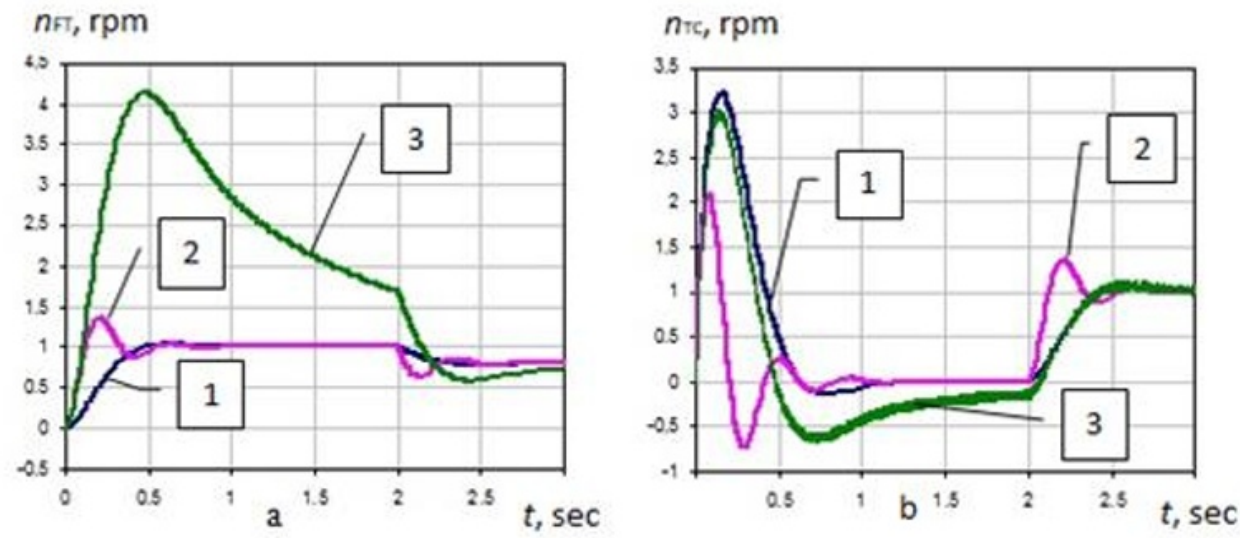

Fig. 7. Transition on $n_{\mathrm{FT}}$ and $n_{\mathrm{TC}}$ with the second matrix $\mathbf{Q}$ : a $-n_{\mathrm{FT}}, \mathrm{b}-n_{\mathrm{TC}} ; 1$ - reference model, 2 - ACS GTU without adaptive control, 3 - ACS GTU with adaptive control. 
The variation of the coefficients of the matrix $\mathbf{Q}$ confirmed the assumption that due to its choice it is possible to improve one adjustable variable at the expense of another. Figure 6 shows the transient process on $n_{\mathrm{FT}}$ and $n_{\mathrm{TC}}$ with the matrix $\mathbf{Q}=\left(\begin{array}{cc}1000 & 0 \\ 0 & 1\end{array}\right)$. Figure 7 - with the matrix $\mathbf{Q}=\left(\begin{array}{ll}1 & 0 \\ 0 & 1\end{array}\right)$. In both cases, $h=100$.

\section{Conclusion}

The use of a second-order dynamic model made it possible, due to the neglect of secondary factors, to investigate the influence of adaptive control parameters on the quality of transients. The following results were obtained by varying the values of $K_{d}, h, d t$.

1) The Euler method can provide a smaller error than the Runge-Kutta method and methods of higher orders.

2) With small deviations of the $K_{d}$ parameters and large $h$, an increase in the control error is several times more significant than with large deviations of the parameters.

3) For small $h$, regulation is better near small deviations of parameters, but worse - with large deviations of parameters.

4) In the matrix $\mathbf{Q}$, the ratio is important, not the absolute values of the elements of the matrix. With the same ratio of matrix elements, the results are the same. Increasing the first element of the matrix $\mathbf{Q}$, we approach the system closed only in the first variable.

5) There is some optimum in the choice of elements of the matrix $\mathbf{Q}$, which provides some best ratio of errors in $x_{1}$ and $x_{2}$.

The reported study was funded by RFBR and Perm region according to the research project № 19-48-590012.

\section{References}

[1] Plis M., Rusinowski H., Predictive, adaptive model of PG 9171E gas turbine unit including control algorithms Energy 126, 247-55 (2017)

[2] Hanachi H., Liu J., Mechefske C., Multi-mode diagnosis of a gas turbine engine using an adaptive neuro-fuzzy system Chinese journal of aeronautics 31, 19 (2017)

[3] Tsai A. , Pezzini P. ,Tucker D., and Bryden K. M., Multiple-Model adaptive control of a hybrid solid oxide fuel cell gas turbine power plant simulator Journal of electrochemical energy conversion and storage 16(3), (2019)

[4] Kavalerov B.V., Bakhirev I.V., Kilin G.A., Adaptive frequency control of a gas turbine unit with a custom model Electrical engineering issue 11, 1-9 (2017)

[5] Kavalerov B.V., Bakhirev I.V. and Basargin Sh. D. The study of the parametric adaptation of the regulator of the gas turbine unit of a $6 \mathrm{MW}$ power plant Fundamental research issue 11-2, 281-285 (2016)
[6] Kavalerov B.V., Bakhirev I.V. and Ziyatdinov I.R. Investigation of an adaptive control algorithm with gas turbine units taking into account the dynamics of a asynchronous engine Fundamental research issue 12-1, 49-57

[7] Kavalerov B. V., Bakhirev I. V., and Kilin G. A., Investigation of the electric power gas turbines rotation frequency adaptive control (Perm: PNIPU press, 142153 (2015)

[8] Kavalerov B. V., and Ziyatdinov I. R., Investigation of the control system with a reference model and parametric adjustment for an electric power gas turbine unit Fundamental research issue 12-6, 11071111 (2015)

[9] Kavalerov B. V., Ziyatdinov I. R., Bakhirev I. V., Investigation of the control system with a reference model and signal setting for an electric power gas turbine unit Fundamental research issue 6-2, 235-240 (2015)

[10] Tyukin I. Yu., Adaptation in nonlinear dynamic systems Synergetics: from the past to the future (St. Petersburg: LKI) 384 (2018)

[11] Ricketts B. E., Modelling of a gas turbine: A precursor to adaptive control. IEE Colloquium on Adaptive Controllers in Practice '97: Digest, is. 176 (1997)

[12] Piltan F., et al. On line Tuning Premise and Consequence FIS: Design Fuzzy Adaptive Fuzzy Sliding Mode Controller Based on Lyaponuv Theory International Journal of Robotics and Automation 2(5), 381-400 (2011)

[13] Gusev Yu. M., TVVD automatic control system with specific fuel consumption optimization Bulletin of Ufa state aviation technical University 15 issue 5(45), (2011)

[14] Skorospeshkin V. N., Adaptive automatic control system Science of science is. 2(21), (2014)

[15] Gurevich O. S., Aviation Gas Turbine Engine Management: Study Guide (Moscow: MAI press) 100 (2001)

[16] Borcov Yu. A., Electromechanical systems with adaptive and model control (Leningrad: Energoatomizdat) 216 (1984)

[17] Basharin A. V., Electric Drive Control: $A$ Textbook for Universities (Leningrad: Energoatomizdat) 392 (1982)

[18] Polulyakh A. I., Lisovin I. G., Kavalerov B. V. and Shigapov A. A., Automating controller tuning for gas-turbine mini power stations in computer testing Automation and Remote Control 75(7), 1330-1336 (2014)

[19] Bakhirev A. V., Adaptive control of a gas turbine plant with a reference model and sigmoidal function Control systems and information technology issue 3.1(61), 118-123 (2015)

[20] Cybenco G., Approximation by superpositions of a sigmoidal function. Mathematics of Control, Signals and System issue 2(4), 303-314 (1989) 